\title{
Reconstructing Higgs boson properties from the LHC and Tevatron data
}

Pier Paolo Giardino, ${ }^{a}$ Kristjan Kannike, ${ }^{b, c}$ Martti Raidal ${ }^{c, d, e}$ and Alessandro Strumia ${ }^{a, c}$

${ }^{a}$ Dipartimento di Fisica, Università di Pisa and INFN,

Polo Fibonacci Largo B. Pontecorvo 3, Pisa, Italy

${ }^{b}$ Theoretical Physics Group, Scuola Normale Superiore and INFN, Piazza dei Cavalieri 7, Pisa, Italy

${ }^{c}$ National Institute of Chemical Physics and Biophysics, Ravala 10, Tallinn, Estonia

${ }^{d}$ CERN, Theory Division, CH-1211 Geneva 23, Switzerland

e Department of Theoretical Physics, Institute of Physics, Tähe 4, University of Tartu, Estonia

E-mail: pierpaolo.giardino@pi.infn.it, Kristjan.Kannike@cern.ch, Martti.Raidal@cern.ch, astrumia@mail.df .unipi.it

ABstract: We perform a phenomenological fit to all ATLAS, CMS, CDF and D0 Higgs boson data available after Moriond 2012. We allow all Higgs boson branching fractions, its couplings to standard model particles, as well as to an hypothetical invisible sector to vary freely, and determine their current favourite values. The standard model Higgs boson with a mass $125 \mathrm{GeV}$ correctly predicts the average observed rate and provides an acceptable global fit to data. However, better fits are obtained by non-standard scenarios that reproduce anomalies in the present data (more $\gamma \gamma$ and less $W W$ signals than expected) such as modified rates of loop processes or partial fermiophobia. We find that present data disfavours Higgs boson invisible decays. We consider implications for the standard model, for supersymmetric and fermiophobic Higgs bosons, for dark matter models, for warped extra-dimensions.

KEYwords: Beyond Standard Model, Standard Model

ARXIV EPRINT: 1203.4254 


\section{Contents}

1 Introduction 1

2 Data and statistical analysis 2

3 Reconstructing the Higgs boson properties 5

3.1 Reconstructing the Higgs boson branching fractions 5

$\begin{array}{lll}3.2 & \text { Reconstructing the Higgs boson invisible width } & 6\end{array}$

3.3 Reconstructing the Higgs boson couplings 8

4 Implications for Higgs boson models $\quad 9$

$\begin{array}{ll}4.1 & \text { The Standard Model } \\ & 10\end{array}$

$\begin{array}{lll}4.2 & \text { Fermiophobia and dysfermiophilia } & 11\end{array}$

$\begin{array}{lll}4.3 \text { Supersymmetry } & 12\end{array}$

$\begin{array}{ll}4.4 \text { Dark matter models } & 12\end{array}$

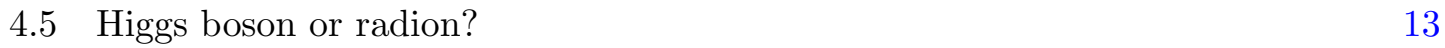

5 Conclusions 14

\section{Introduction}

Identifying the mechanism of electroweak symmetry breaking is the main goal of the Large Hadron Collider (LHC). In the standard model (SM) the electroweak symmetry is broken due to the existence of an elementary scalar particle - the Higgs boson [1-5]. Based on data collected in 2011, both the ATLAS and CMS experiments at the LHC published results of their searches for the SM-like Higgs boson that, yet inconclusively, support its existence with a mass $m_{h} \approx 125 \mathrm{GeV}$ [6-9]. Those results have been recently updated at the Moriond 2012 conference, where all the Tevatron and LHC collaborations presented their updated Higgs boson searches as well as some new results. The combined Tevatron analysis of all collected data confirms the LHC excess around $125 \mathrm{GeV}$ in the $h \rightarrow b \bar{b}$ channel at $2.6 \sigma$ level; CMS presented an improved $\gamma \gamma$ analysis; ATLAS presented new $W W^{*}, b \bar{b}$ and $\tau \bar{\tau}$ searches with full 2011 luminosity. Furthermore, both the ATLAS and CMS experiments showed results of searches for a fermiophobic (FP) Higgs boson in the $h \rightarrow \gamma \gamma$ channel that both show a positive hint around $125 \mathrm{GeV}$ with local significances about $3 \sigma$. This excess is consistent with the total inclusive $\gamma \gamma$ rate observed by the LHC [10].

Accidentally, $m_{h} \approx 125 \mathrm{GeV}$ is a particularly fortunate value for the LHC, because, according to the SM predictions, various Higgs boson search channels are measurable. Those arise from a combination of SM Higgs boson branching fractions [11, 12]

$$
\begin{aligned}
& \mathrm{BR}(h \rightarrow b \bar{b})=58 \%, \quad \operatorname{BR}\left(h \rightarrow W W^{*}\right)=21.6 \%, \quad \operatorname{BR}\left(h \rightarrow \tau^{+} \tau^{-}\right)=6.4 \%, \\
& \operatorname{BR}\left(h \rightarrow Z Z^{*}\right)=2.7 \%, \quad \operatorname{BR}(h \rightarrow g g)=8.5 \%, \quad \operatorname{BR}(h \rightarrow \gamma \gamma)=0.22 \%,
\end{aligned}
$$


and production mechanisms with cross sections $[13,14]$

$$
\begin{aligned}
\sigma(p p \rightarrow h) & =(15.3 \pm 2.6) \mathrm{pb}, & \sigma(p p \rightarrow j j h) & =1.2 \mathrm{pb}, \\
\sigma(p p \rightarrow W h) & =0.57 \mathrm{pb}, & \sigma(p p \rightarrow Z h) & =0.32 \mathrm{pb},
\end{aligned}
$$

named gluon-gluon fusion $(g g \rightarrow h)$, vector-boson fusion (VBF) and associated production with $W$ and $Z$ bosons (Vh). Because different search categories are sensitive to different Higgs boson couplings, the LHC can study the properties of a Higgs boson with $m_{h} \approx$ $125 \mathrm{GeV}$ and test if it follows the SM predictions or is affected by new physics.

With the presently collected statistics none of the search channels alone is sensitive to the SM Higgs boson nor are the combined results of Tevatron, ATLAS and CMS statistically conclusive. Therefore one expects large statistical fluctuations of the expected signal in all the search channels. Indeed, all measured LHC $\gamma \gamma$ rates, dominated by the new results in the VBF category, have central values above the SM prediction while all the $W W^{*}$ rates have central values consistently below the SM prediction. On the one hand, those anomalies may be statistical fluctuations. On the other hand, they may signal new physics beyond the SM. From a theoretical point of view, reconstructing the Higgs boson properties is an important way to address the main issue that LHC can clarify: is there a natural reason behind the the smallness of the weak scale, $m_{h} \ll M_{\mathrm{Pl}}$ ? Indeed, if the weak scale is naturally small, one expects that the new physics that cuts off the top loop contribution to $m_{h}^{2}$ (such as light stops at the weak scale in supersymmetric models) also affects the $g g \rightarrow h$ and $h \rightarrow \gamma \gamma$ rates. Therefore a global study of all the Higgs boson collider data obtained so far is necessary to test the SM and to discriminate between different new physics scenarios in the Higgs sector.

In this work we study the collider data collected so far in Tevatron and the LHC in order to derive Higgs boson properties. Some authors discussed how to perform Higgs fit $[15,16]$, and actual fits of recent LHC data were performed in [17-19]. We improve on previous fits by including the new data presented in the Moriond 2012 conference, and by performing more general fits that cover a wider spectrum of new physics models. To achieve this goal we allow all the Higgs boson couplings to deviate independently from their SM values. We also allow for an additional Higgs boson invisible width, possibly due to decays into the dark matter. Anomalous features are dominated by the new results presented in Moriond 2012, disfavouring the SM compared to the previous fits and motivating new physics scenarios. We discuss implications of our results in the context of different models. More LHC data is needed to discriminate between those scenarios.

The paper is organised as follows. In section 2 we describe the existing experimental results and the statistical procedure we adopt. In section 3 we perform the fits to data. In section 4 we discuss implications of our results on different models. We conclude in section 5 .

\section{Data and statistical analysis}

The experimental collaborations measure rates of Higgs boson signals $R$. Their results could be fully encoded in a likelihood $\mathcal{L}\left(R, m_{h}\right)$, but only a limited amount of information 
is reported by the experiments. All collaborations report the upper bounds on rates at $95 \%$ C.L., $R_{\text {observed }}$, and the expected upper bound at $95 \%$ C.L. in absence of a Higgs boson signal, $R_{\text {expected }}$, as function of the Higgs boson mass $m_{h}$. Given that information, our statistical analyses follows the one outlined in ref. [20]. Assuming that the $\chi^{2}=-2 \ln \mathcal{L}$ has a Gaussian form in $R$,

$$
\chi^{2}=(R-\mu)^{2} / \sigma^{2},
$$

these two experimental informations allow one to extract the mean $\mu$ and the standard deviation $\sigma$,

$$
\mu=R_{\text {observed }}-R_{\text {expected }}, \quad \sigma=\frac{R_{\text {expected }}}{1.96}
$$

where 1.96 arises because $95 \%$ confidence level corresponds to about 2 standard deviations. The Gaussian approximation by construction agrees with the full result at this value of $R$, but away from it the approximation may be not accurate for channels that presently have a low number of events (such as $h \rightarrow Z Z^{*} \rightarrow 4 \ell$ ). We are aware of this fact, but at the moment it is difficult to do better using the available data. We verified that our procedure gives similar results as the refined procedure in [17] and that our procedure agrees better with present values of $\mu \pm \sigma$, when reported by experiments at $m_{h}=125 \mathrm{GeV}$.

We do not discuss theoretical uncertainties separately because they have already been taken into account in the data we use. We also neglect correlations of uncertainties among different measurements (e.g. uncertainties on luminosity and on the SM prediction, at the $\pm 15 \%$ level and therefore subdominant with respect to present experimental uncertainties) and approximate the full $\chi^{2}$ with

$$
\chi^{2}=\sum_{i} \frac{\left(R_{i}-\mu_{i}\right)^{2}}{\sigma_{i}^{2}},
$$

where the sum runs over all measured Higgs boson rates $i$. In the present stage of experimental accuracy such a simplified statistical framework captures the main features in data and allows us to study general properties of the data, that is the purpose of this work.

We consider all available Higgs boson data reported at the Moriond 2012 conference and before:

1. The $p \bar{p} \rightarrow V h \rightarrow V b \bar{b}$ rate measured by CDF, D0 [21, 22] and the related $p p \rightarrow V h \rightarrow$ $V b \bar{b}$ rate measured by CMS and ATLAS [23, 24].

2. The $p p \rightarrow j j h \rightarrow j j W W$ rate measured by CMS [25].

3. The $h \rightarrow W W \rightarrow 2 \ell 2 \nu$ rates measured by CMS and ATLAS [26-28].

4. The $h \rightarrow Z Z \rightarrow 4 \ell$ rates measured by CMS and ATLAS [28, 29].

5. The $h \rightarrow \gamma \gamma$ rates measured by ATLAS and CMS [30-32], and CDF, D0 [33].

6. In the context of fermiophobic Higgs boson searches, CMS measured the $p p \rightarrow j j h \rightarrow$ $j j \gamma \gamma$ rate $[34,35]$ where the $j j$ tagging is added to select Higgs boson produced via the VBF process. Indeed, the cuts performed by $\mathrm{CMS}\left(m_{j j}>350 \mathrm{GeV}, p_{T j_{1}}>\right.$ 

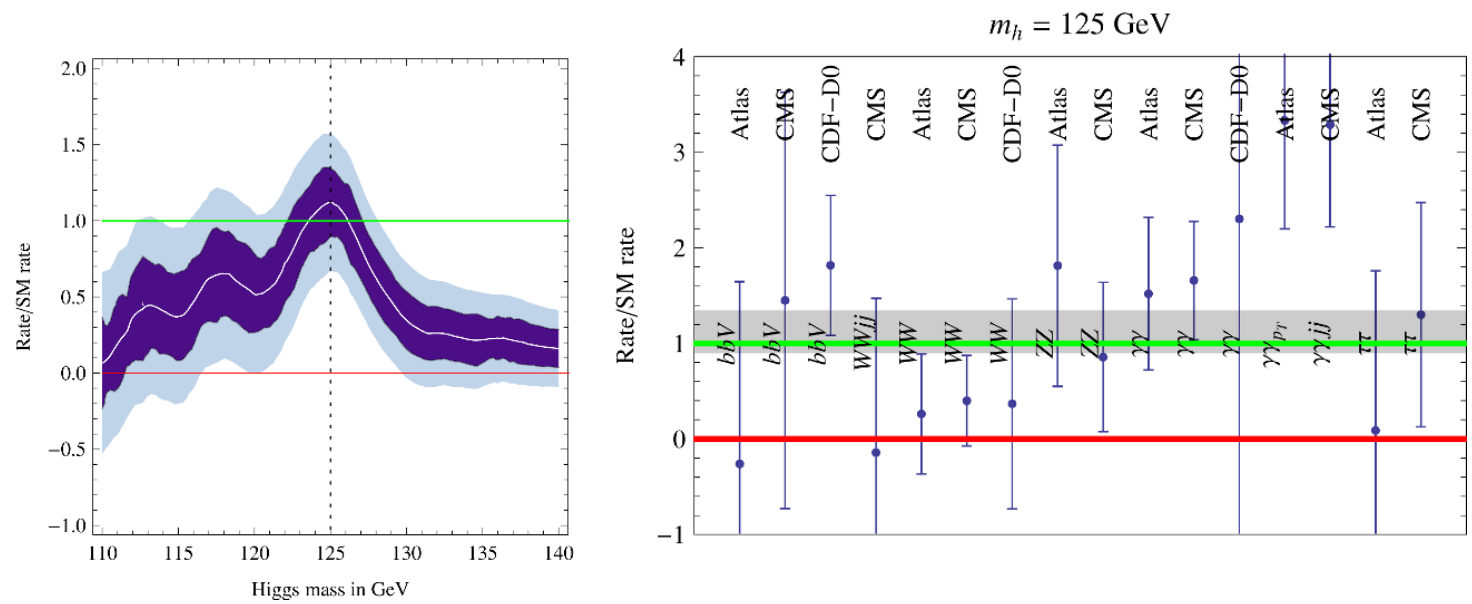

Figure 1. Left: the Higgs boson rate favoured at $1 \sigma$ (dark blue) and $2 \sigma$ (light blue) in a global SM fit as function of the Higgs boson mass. Right: assuming $m_{h}=125 \mathrm{GeV}$, we show the measured Higgs boson rates at ATLAS, CMS, CDF, D0 and their average (horizontal gray band at $\pm 1 \sigma$ ). Here 0 (red line) corresponds to no Higgs boson, 1 (green line) to the SM Higgs boson.

$\left.30 \mathrm{GeV}, p_{T j_{2}}>20 \mathrm{GeV}\right)$ significantly reduce the $g g \rightarrow h$ contribution, such that we estimate that reinterpreting this experimental result in a general context, it is roughly a measurement of

$$
[0.033 \sigma(p p \rightarrow h)+\sigma(p p \rightarrow j j h)] \times \mathrm{BR}(h \rightarrow \gamma \gamma)
$$

with the result

$$
\frac{\text { observed rate }}{\text { SM rate }}=3.3 \pm 1.1 \text { for } m_{h}=125 \mathrm{GeV} \text {. }
$$

7. In the context of fermiophobic Higgs boson searches, ATLAS measured the $p p \rightarrow$ $h X \rightarrow \gamma \gamma X$ rate with a high cut $p_{T h}>40 \mathrm{GeV}$ on the Higgs boson transverse momentum [36] (we are oversimplifying by omitting several secondary issues). This cut allows to suppress the $g g \rightarrow h$ production process, while keeping most of the signal in the VBF and associate production mechanisms. To see how much $g g \rightarrow h$ is suppressed we allowed for additional QCD jets performing simulations with the Pythia [37] and MadGraph [38] codes. We find that this experimental result can be re-interpreted in a general context as a measurement of

$$
[0.3 \sigma(p p \rightarrow h)+\sigma(p p \rightarrow W h, Z h, j j h)] \times \mathrm{BR}(h \rightarrow \gamma \gamma)
$$

with the result

$$
\frac{\text { observed rate }}{\text { SM rate }}=3.3 \pm 1.1 \quad \text { for } m_{h}=125 \mathrm{GeV} \text {. }
$$

8. The $h \rightarrow \tau \tau$ rate as measured by CMS and ATLAS [39, 40]. 
In the left panel of figure 1 we show our approximated combination of all Higgs boson data. Higgs boson masses around $125 \mathrm{GeV}$ are favoured by the rate, and some $Z Z$ and $\gamma \gamma$ events (which have little statistical power in fixing the rates but large resolution in $m_{h}$ ) favor $m_{h}=125 \mathrm{GeV}$, the value that we will adopt in the rest of the paper.

Notice that, provided that present hints are really due to the Higgs boson, its mass is already known so precisely that the uncertainty on $m_{h}$ has negligible effect on the fit, because the expected SM rates have only small variations in the favored range between 124 and $126 \mathrm{GeV}$. On the other hand, if the present excess in $\gamma \gamma$ distributions around $125 \mathrm{GeV}$ will turn out to be statistical fluctuations, then the $\gamma \gamma$ rate will change significantly with respect to what assumed in our fits on the basis of present data.

Assuming $m_{h}=125 \mathrm{GeV}$, we summarise all data in the right panel of figure 1 together with their $1 \sigma$ error-bars, as derived by us following the above-described statistical procedure. The horizontal green line in the right panel of figure 1 is the SM prediction, and the horizontal red line is the background-only rate expected in the absence of a Higgs boson. The grey band shows the $\pm 1 \sigma$ range for the weighted average of all data. It lies along the SM prediction. Furthermore, the global $\chi^{2}$ of the SM fit is 17 for 15 dof.

However, it is interesting to split data into three categories according to the final states and compute the average for each one of them:

$$
\frac{\text { observed rate }}{\text { SM rate }}=\left\{\begin{array}{l}
2.1 \pm 0.5 \text { photons } \\
0.5 \pm 0.3 \text { vectors: } W \text { and } Z \\
1.3 \pm 0.5 \text { fermions: } b \text { and } \tau
\end{array} .\right.
$$

This shows the main anomalous features in current measurements. First, the $\gamma \gamma$ channels exhibit some excess, mainly driven by the vector boson fusion data presented at the Moriond 2012 conference. Second, there is a deficit in the vector channels. Finally, the average rate of fermionic channels lies along the SM prediction; here the new Tevatron combination for $h \rightarrow b \bar{b}$ plays an important rôle.

\section{Reconstructing the Higgs boson properties}

\subsection{Reconstructing the Higgs boson branching fractions}

The Higgs boson observables that can be most easily affected by new physics contributions are those that occur at loop level, the $h \rightarrow \gamma \gamma, h \rightarrow g g$ and $g g \rightarrow h$ rates. Because the latter two are related via CP, we use a common notation $h \leftrightarrow g g$ to indicate both of them simultaneously. Those loop level processes are particularly relevant for the LHC Higgs boson searches because $\gamma \gamma$ is the cleanest final state, and because $g g \rightarrow h$ is the dominant Higgs boson production mechanism. The left panel of figure 3 shows, as yellow contours with solid borders, the $1 \sigma$ and $2 \sigma$ ranges of a global fit to these two quantities in units of their SM predictions. The best fit corresponds to

$$
\frac{\mathrm{BR}(h \leftrightarrow g g)}{\mathrm{BR}(h \rightarrow g g)_{\mathrm{SM}}} \approx 0.3, \quad \frac{\mathrm{BR}(h \rightarrow \gamma \gamma)}{\mathrm{BR}(h \rightarrow \gamma \gamma)_{\mathrm{SM}}} \approx 4
$$




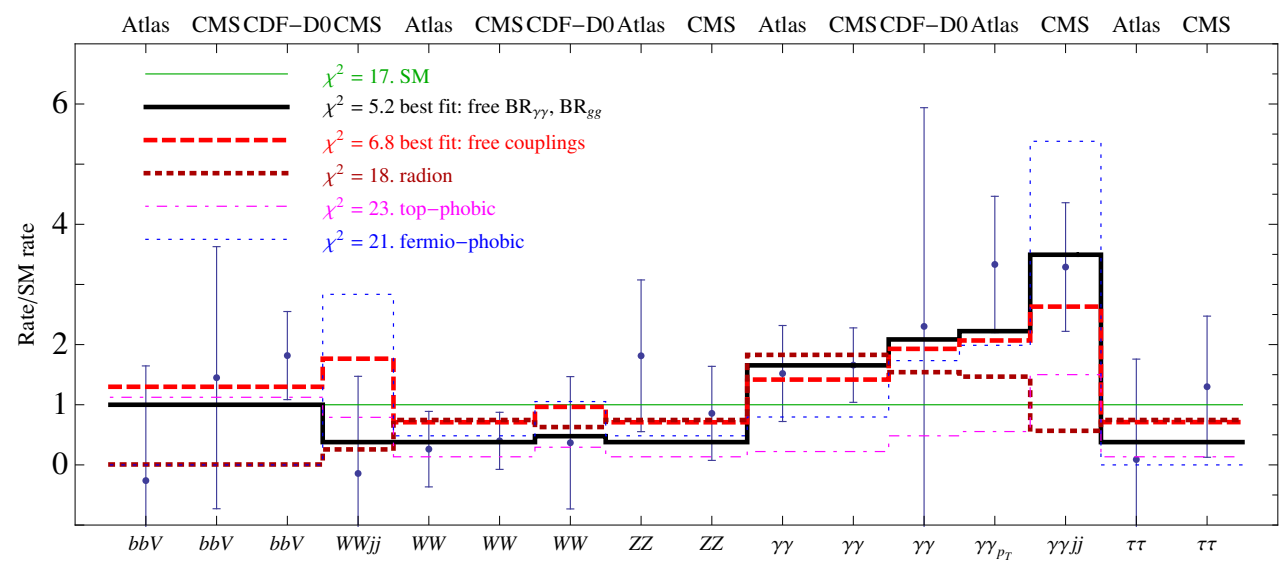

Figure 2. Predictions for the Higgs boson rates in different scenarios: SM, free branching ratios of loop processes, free couplings, radion, top-phobic and fermiophobic, defined via eqs. (3.4), (3.5), (4.5).

that shows a significant deviation from the SM prediction - the first number allows to best fit the reduced $W W^{*}$ rates, and the second number allows to fit the enhanced $\gamma \gamma$ rates, in agreement with eq. (2.8). The $\chi^{2}$ of the global fit is significantly lower with respect to $\mathrm{SM}$, decreasing from 17 (for 15 dof within the SM) to 5.2 (for 13 dof in this more general fit). The black thick line in figure 2 shows the best-fit predictions for the various measured rates, allowing to see how the fit is improved.

The gray region with dotted contours in figure 3 show the fit obtained omitting the $\gamma \gamma$ rates with cuts dedicated to vector-boson-fusion production (items 6 and 7 in the list above). In the latter case the agreement with the SM is improved showing that the such data category plays an important rôle in the fit.

\subsection{Reconstructing the Higgs boson invisible width}

New physics can easily give a large effect providing an extra invisible [41-43] Higgs boson decay channel, for example into dark matter particles [43-45, 45-59]. Alternatively, the effective operator $\left|\partial_{\mu} H^{\dagger} H\right|^{2}$ similarly has the effect of rescaling all rates by a common factor $[60-63]$.

In the SM the total Higgs boson width is predicted to be $\Gamma(h)_{\mathrm{SM}} \approx 4.0 \mathrm{MeV}$ at $m_{h}=$ $125 \mathrm{GeV}$, too small to be measured directly.

It is well known that measuring the Higgs boson total width at the LHC requires additional assumptions $[15,16]$. Let us explain how present data can probe the Higgs boson width, without directly measuring it. In view of CP invariance we can assume the equality of $g g \rightarrow h$ and $h \rightarrow g g$ amplitudes, that we collectively denote as $h \leftrightarrow g g$. The gluon fusion production rate is then proportional to $\Gamma(g g \rightarrow h)$ as given by the well known Breit-Wigner formula

$$
\sigma(g g \rightarrow h)=\frac{\pi}{8} \frac{\Gamma(h \rightarrow g g) \Gamma(h)}{\left(s-m_{h}^{2}\right)^{2}+m_{h}^{2} \Gamma(h)^{2}} \stackrel{\Gamma(h) \ll m_{h}}{\simeq} \frac{\pi^{2}}{8 m_{h}} \Gamma(h \rightarrow g g) \delta\left(s-m_{h}^{2}\right) .
$$



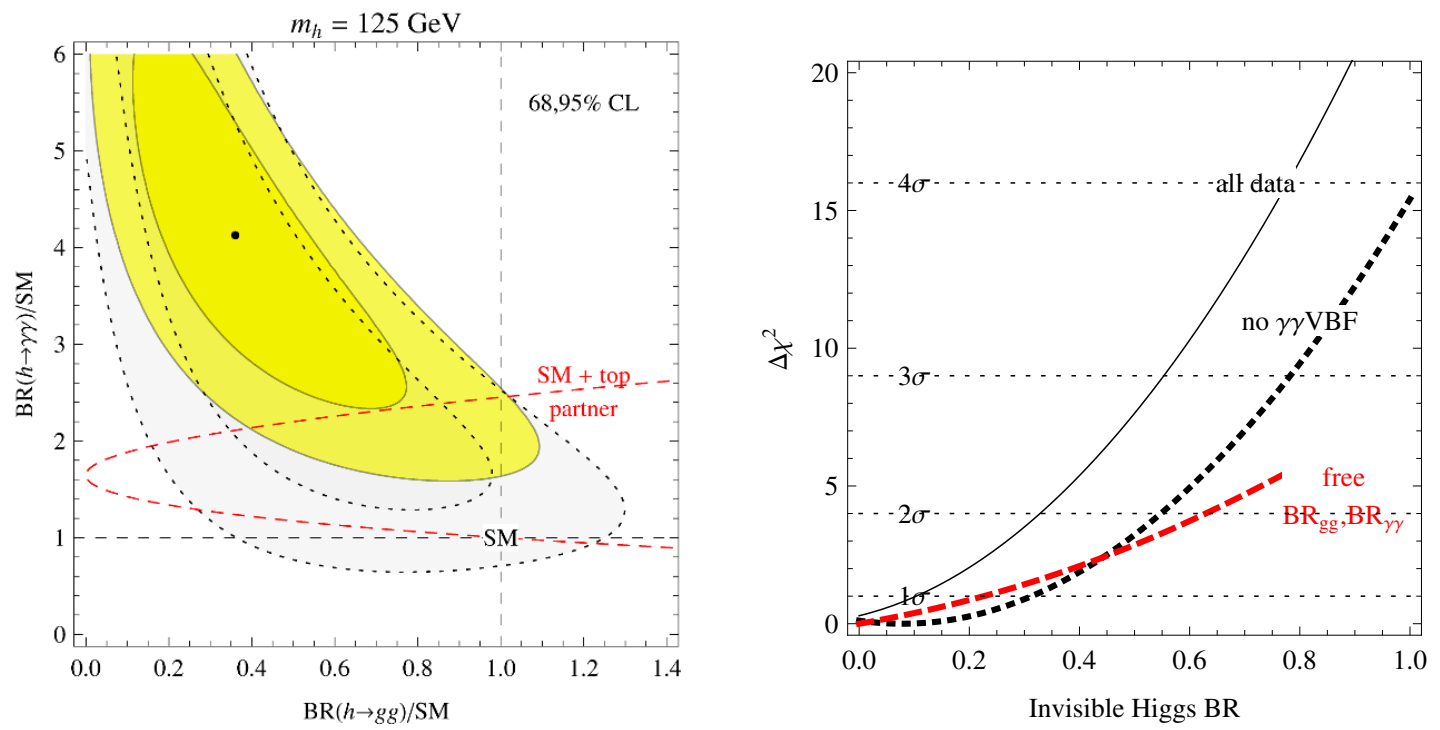

Figure 3. Left: fit for the Higgs boson branching fraction to photons and gluons. In yellow with continuous contour-lines: global fit. In gray with dotted contour-lines: the fermiophobic Higgs boson searches are excluded from the data-set. Red dashed curve: the possible effect of extra top partners, such as the stops. Right: fits for the invisible Higgs boson branching fraction, under different model assumptions, as explained in section 3.2.

Then, one partial decay width can be reconstructed by data. By performing a global fit to the Higgs boson branching ratios in the context of theories where the decay widths are related we can reconstruct the total Higgs boson width. Of course this is based on theoretical assumptions, but the result gets significantly different only in highly deviant models, e.g. in models where the Higgs boson predominantly decays into light quarks (a decay mode not probed by present data).

In order to emphasise the mild model-dependence of this fitting procedure we perform three fits under different assumptions. We show our results $\left(\chi^{2}\right.$ as function of the invisible branching ratio) in the right panel of figure 3 .

i) First, we perform a global fit of all data assuming the SM plus an additional invisible decay width, obtaining

$$
\mathrm{BR}(h \rightarrow \text { invisible })=-0.1 \pm 0.23 ;
$$

ii) Next, we weaken the theoretical assumptions: we keep the $h \leftrightarrow g g$ and the $h \rightarrow \gamma \gamma$ rates as free parameters, and marginalise with respect to them (red dashed curve). We see that, even without assuming the SM prediction for $h \leftrightarrow g g$, a (weakened) bound on the Higgs boson invisible width can still be derived from present data; the best fit value becomes positive, but again the preference is not statistically significant.

iii) Finally, we repeat the fit in i), but ignoring the data for $\gamma \gamma$ from the vector boson fusion channels, obtaining a weaker bound (dotted curve). 
Adding an invisible Higgs boson width has the effect of suppressing all observed rates and, according to our fit, this is not favoured by present data.

\subsection{Reconstructing the Higgs boson couplings}

In this subsection we extract from data the Higgs boson couplings to vectors and fermions, in order to see if they agree with the SM predictions. Trying to be as general as possible in describing the Higgs boson couplings, we proceed phenomenologically extracting from data the following parameters:

$$
R_{W}=\frac{g_{W}}{g_{\mathrm{W}}^{\mathrm{SM}}}, \quad R_{Z}=\frac{g_{Z}}{g_{\mathrm{V}}^{\mathrm{SM}}}, \quad R_{t}=\frac{y_{t}}{y_{t}^{\mathrm{SM}}}, \quad R_{b}=\frac{y_{b}}{y_{b}^{\mathrm{SM}}}, \quad R_{\tau}=\frac{y_{\tau}}{y_{\tau}^{\mathrm{SM}}},
$$

where $g_{W}$ is the $W W h$ coupling; $g_{Z}$ is the $Z Z h$ coupling, $y_{t}$ the top Yukawa coupling, $y_{b}$ the bottom Yukawa coupling and $y_{\tau}$ the tau Yukawa coupling. All models considered in this work and presented in figure 2 are defined via eq. (3.4). The SM corresponds to $R_{i}=1$ for all the couplings. These parameter $R_{i}$ have the following effects:

- the partonic cross sections for $g g \rightarrow h$ and for $g g \rightarrow t \bar{t} h$ get rescaled by $R_{t}^{2}$;

- the partonic cross sections for $q \bar{q} \rightarrow q \bar{q} h$ and for $q \bar{q} \rightarrow V h$ get rescaled by $R_{V}^{2}$;

- the decay widths $h \rightarrow V V^{*}$ get rescaled by $R_{V}^{2}$ where $V=\{W, Z\}$;

- the decay widhts $h \rightarrow f \bar{f}$ get rescaled by $R_{f}^{2}$ where $f=\{b, \tau, \ldots\}$;

- the decay width $h \rightarrow \gamma \gamma$, arising from the interference of one-loop diagrams mediated by the top and by the $W$, gets rescaled by $\left(1.28 R_{W}-0.28 R_{t}\right)^{2}$ for $m_{h}=125 \mathrm{GeV}$;

- similarly the decay width $h \rightarrow Z \gamma$ (not yet measured) gets rescaled by $\left(1.05 R_{Z}-\right.$ $\left.0.05 R_{t}\right)^{2}$.

A simplifying case considered in previous analyses [17-19] is a common rescaling factor $a$ for Higgs boson coupling to vectors and a common rescaling factor $c$ for Higgs boson coupling to fermions:

$$
a=R_{V} \equiv R_{W}=R_{Z}, \quad c=R_{t}=R_{b}=R_{\tau} .
$$

We show in the left panel of figure 4 the resulting fit (continuous yellow contours). For comparison the dashed contours show the result obtained ignoring the $\gamma \gamma j j$ data from CMS and ATLAS, as is also done in figure 3. This allows to compare our results with the ones of previous analyses [17-19] (although some other data has also been modified and added by experiments). Our results essentially agree, up to the difference due to our use of more recent data.

We see that a negative $R_{t} R_{W}<0$ is favoured because it implies a constructive interference between the top quark and $W$ boson loops in the decays $h \rightarrow \gamma \gamma$ increasing the corresponding rates. Notice that the new data prefers suppression of the $W W^{*}$ rates via suppression of the $g g \rightarrow h$ cross section, while the Higgs boson coupling to vectors 

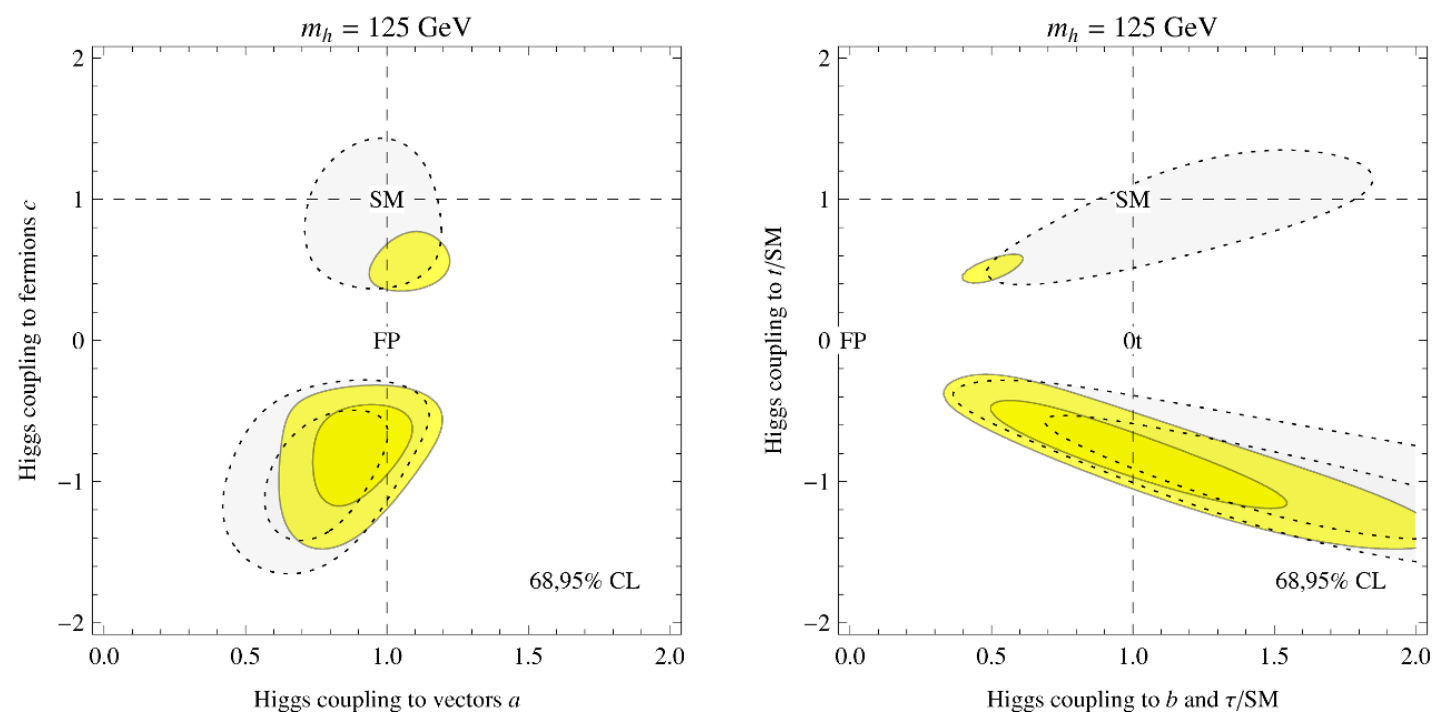

Figure 4. Left: fit of the Higgs boson couplings assuming common rescaling factors $a$ and $c$ with respect to the SM prediction for vector bosons and fermions, respectively. Right: fit to the $t$-quark and to $b$-quark and $\tau$-lepton Yukawa couplings assuming the SM couplings to gauge bosons. The best fit presently lies somehow away from the SM prediction, indicated in the figures as 'SM'. The point marked as ' $\mathrm{FP}$ ' is the fermiophobic case, and ' $0 \mathrm{t}$ ' denotes the top-phobic case. Negative values of the top Yukawa coupling are preferred because lead of an enhancement of $h \rightarrow \gamma \gamma$.

can be somewhat larger than without VBF data. Notice also that the SM point $(1,1)$ is disfavoured beyond $2 \sigma$.

In the right panel of figure 4 we assume the SM values for the Higgs boson gauge couplings $\left(R_{W}=R_{Z}=1\right)$ and present a fit to the Yukawa couplings $R_{t}$ and $R_{b}=R_{\tau}$. We, again, see that $R_{t}<0$ is somehow favoured and the SM is disfavoured. The two branches approach the pure fermiophobic point $(0,0)$, denoted by FP in figure 4 , but pure fermiophobia is disfavoured by the fit.

In figure 5 we consider the most general case where we allow all 4 parameters $R_{W}=$ $R_{Z}, R_{t}, R_{b}, R_{\tau}$ to vary and show the favoured regions for the pairs $R_{V}, R_{t}$ (left) and $R_{b}, R_{\tau}$ (right) marginalised over the remaining two parameters. The main features of this global fit remain the same as in previous cases: $R_{t} R_{W}<0$ is favoured and $R_{W}, R_{b}$ and $R_{\tau}$ are constrained to be around their SM values of 1 . Figure 2 shows the best fits (red dashed lines), both allowing for negative Yukawas (thick line) and restricting all Yukawas to be positive, as in the SM (thin line).

\section{Implications for Higgs boson models}

In order to interpret our general results presented in figures 1-5 in the context of any particular model of Higgs boson, two logical possibilities arise. First, all the present anomalies in data, listed in eq. (2.8), could be just statistical fluctuations. Second, the emerging pattern in eq. (2.8) could be real and signal new physics beyond the SM in the Higgs sector. Intermediate possibilities are of course possible. In order to discriminate between 

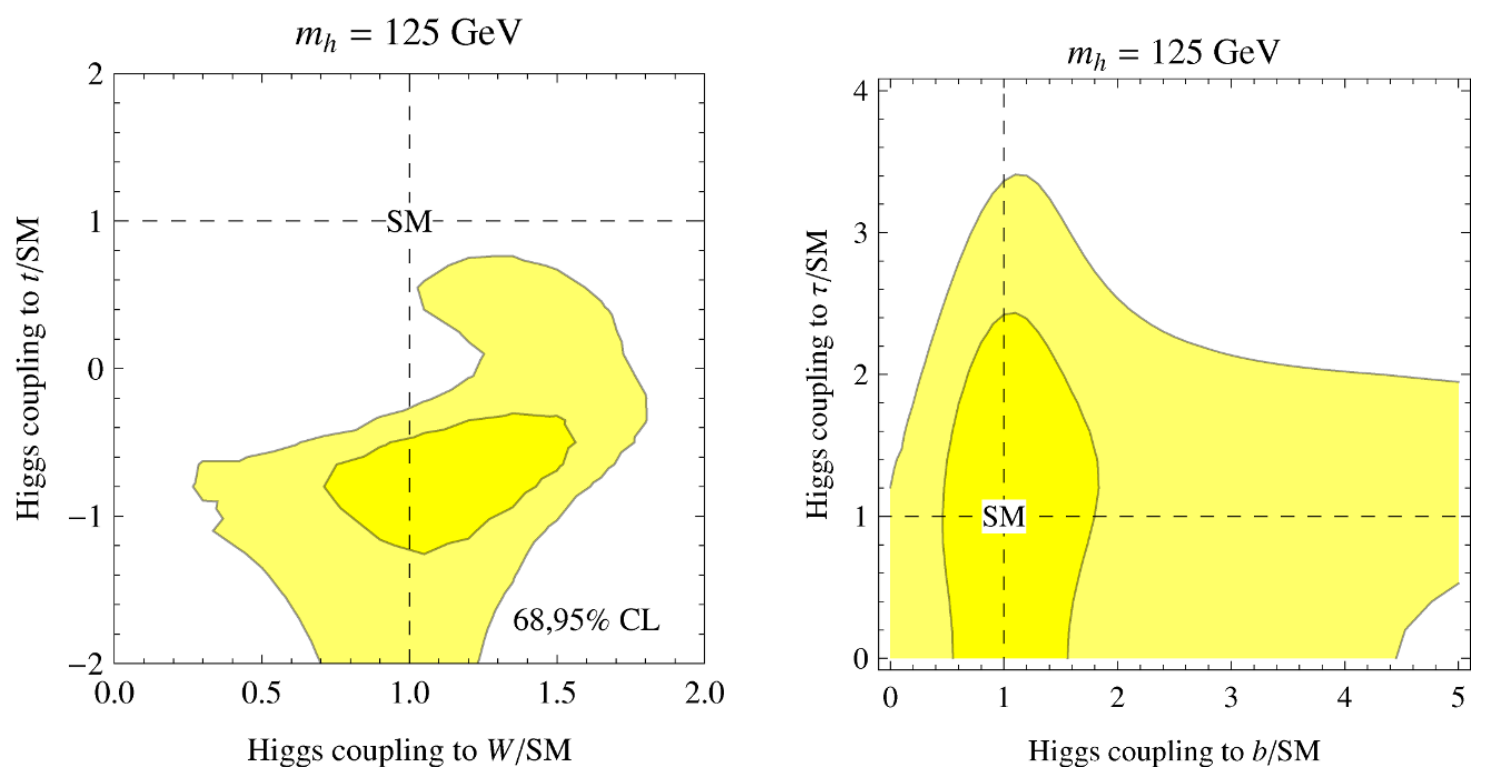

Figure 5. Global fit for the Higgs boson couplings to vectors, to the $t$-quark, to the $b$-quark, to the $\tau$ lepton. All these couplings are freely varied and in each panel we show the $\chi^{2}$ as function of the parameters indicated on the axes, marginalised with respect to all other parameters. We again assume $m_{h}=125 \mathrm{GeV}$ and find that the best fit presently lies somehow away from the SM prediction, indicated in the figures as 'SM'.

these possibilities, we present in figure 2 the predictions channel by channel of some particular scenarios that we studied in figures $3-5$ for collider searches. The best fit $\chi^{2}$ of those scenarios is also presented in the figure in order to compare different scenarios with each other.

\subsection{The Standard Model}

Naturally, the reference model for all comparisons in the previous sections is the SM. After fixing the Higgs boson mass to the best fit value $m_{h}=125 \mathrm{GeV}$, the SM does not have any free parameter left to vary. Therefore all the anomalies in the present data must be statistical fluctuations and disappear with more statistics. This interpretation is supported by the fact that the average of all data agrees with the SM prediction, as seen in figure 2, and the global $\chi^{2}$ is good: 17 for 15 dof (we recall that with $n \gg 1$ degrees of freedom one expects $\chi^{2}=n \pm \sqrt{n}$ ).

On the other hand, our best fit (black curve in figure 2) has a significantly lower $\chi^{2}=5.5$ for 13 dof: a bigger reduction than what is typically obtained by adding two extra parameters (one expects $\Delta \chi^{2}=-\Delta n \pm \sqrt{\Delta n}$ when adding $\Delta n \gg 1$ parameters). The SM is disfavoured by the $\Delta \chi^{2}$ test at more than $95 \%$ CL in this particular context, but of course we added the two parameters that allow to fit the two most apparent anomalies in the data, the $\gamma \gamma$ excess and the $W W^{*}$ deficit, as illustrated in figure 2 .

We recall that the $\chi^{2}$ test and the $\Delta \chi^{2}$ tests are different statistical tools, based on different assumptions and with different statistical powers (see appendix B of [64]). 
The present situation with a good $\chi^{2}$ but a poor $\Delta \chi^{2}$ is ambiguous but it is not selfcontradictory. Only more data will tell if this is a trend, or if we are just fitting a statistical fluctuation.

\subsection{Fermiophobia and dysfermiophilia}

Figure 2 shows predictions for different fermiophobic [10,65-74] scenarios. While bottomphobic Higgs boson is excluded by our fits, top-phobic or pure fermiophobic Higgs boson (with exactly vanishing Yukawa couplings) provide acceptable fits, of quality almost as good as the SM fit, despite that their predictions are significantly different. The pure fermiophobic model captures the features of data qualitatively correctly but predicts larger signal rates than is observed in the LHC, especially in the $W W^{*}+j j$ channel. In addition, the fermiophobic fit suffers from the $h \rightarrow b \bar{b}$ signal claimed by Tevatron and CMS.

The agreement of the fermiophobic Higgs boson with data can be improved by allowing a moderate small additional Higgs boson branching fraction, because this allows to reduce the too large prediction for the $\gamma \gamma j j$ rate [10], which is very sensitive to the precise value of the Higgs boson mass and width. In fermiophobic models such small Yukawa couplings can be generated via quantum effects [72-74]. We note that fermiophobia lowers the vacuum stability bound on the Higgs boson mass, allowing $125 \mathrm{GeV}$ Higgs boson to be consistent with no new physics below Planck scale.

Our fits in figures $4-5$ show that reducing some or all of the SM Yukawa couplings allows to again significantly improve the global fit compared to the SM, down to $\chi^{2} \approx 7$. The main feature of the improved fit is $y_{t} \approx-0.7 y_{t}^{\mathrm{SM}}$, because this allows to enhance the $h \rightarrow \gamma \gamma$ rate and reduce the $g g \leftrightarrow h$ rate. Admittedly, a 'wrong' Yukawa coupling to the top and to the other fermions (dysfermiophilia) is an even more serious pathology than fermiophobia.

Various theoretical frameworks easily lead to modified Higgs boson couplings at moderate level. In models with more than one Higgs multiplet the Yukawa couplings of the light Higgs boson can be non-standard [75, 76] (this is what can happen also in supersymmetric models). Alternatively, in models where SM fermions mix with extra fermions at the weak scale, integrating out the extra fermions, their effects get encoded in effective operators of the form $\bar{f} f H H^{\dagger} H$, that lead to modified Higgs boson couplings to the SM fermions $f[77,78]$. Such operators also arise in composite Higgs boson models.

In models where the Higgs boson is a composite particle one generically expects that Higgs boson couplings get modified by form factors, approximated at low energy by effective operators [60-63]. This is the framework considered in the fits of refs. [17, 19]. In this kind of models, the rescaled SM expressions for these rates that we assumed remain valid even when new physics is so large [79-81]. The Higgs boson coupling to $W, Z$ vectors can be easily reduced by mixing the Higgs boson with other scalars; a good fit to electroweak precision data then demands that the extra scalars are not much heavier than the Higgs boson. Increasing the Higgs boson gauge couplings is theoretically more challenging [82]. 


\subsection{Supersymmetry}

Supersymmetric theories that attempt to solve the naturalness problem of the electroweak scale have been stringently constrained by the LHC direct searches as well as by the Higgs boson results [58, 83-113]. Within the MSSM one needs light and strongly mixed stops, and there are two main modifications of Higgs physics.

First, light stops modify the predictions and for the $h \rightarrow \gamma \gamma$ and $g g \leftrightarrow h$ rates [114]. In practice their extra loop effect is described by a deviation of our parameter $R_{t}$ from one:

$$
R_{t}=1+\frac{m_{t}^{2}}{4}\left[\frac{1}{m_{\tilde{t}_{1}}^{2}}+\frac{1}{m_{\tilde{t}_{2}}^{2}}-\frac{\left(A_{t}-\mu / \tan \beta\right)^{2}}{m_{\tilde{t}_{1}}^{2} m_{\tilde{t}_{2}}^{2}}\right],
$$

at leading order in the limit $m_{\tilde{t}_{1,2}} \gg m_{t}$ [18]. We see that the sign of the new effect is not fixed and can be negative in the presence of strong stop mixing.

The red dashed curve in the left panel of figure 3 shows how these rates are affected by $R_{t}$ (this applies not only to stops, but also to any extra particle with same gauge quantum numbers as the top, such as heavy top partners in little-Higgs models). $R_{t}=1$ corresponds to the SM, and $R_{t}=0$ to the total suppression of $g g \leftrightarrow h$. A $R_{t}<1$ increases the $h \rightarrow \gamma \gamma$ rate, but only mildly because this rate is dominated by the $W$ loop. The red dashed curve enters in the best-fit region when $R_{t} \approx-1.7$, a situation that cannot be achieved in view of bounds on the stop mass. We recall that such bounds are extremely model-dependent, because the signature depends on the unknown stop decay modes, and the production depends on the unknown gluino mass. For example, in gauge-mediated SUSY breaking the lower bound is $310 \mathrm{GeV}$ [115]. If we assume $m_{\tilde{t}_{1}} \approx m_{\tilde{g}}$, the bound on the stop mass is around $900 \mathrm{GeV}$ [116-120], assuming that gluino decays always via a sbottom $\tilde{b}$ into $b N_{1}$ with a neutralino mass $m_{N_{1}}<150-300 \mathrm{GeV}$. The bound on $m_{\tilde{t}}$ gets about $200 \mathrm{GeV}$ lower if the gluino decays fully via a stop.

Second, due to the presence of two Higgs doublets $H_{1}$ and $H_{2}$, one has modification of the Higgs boson couplings at tree level. Our $R$ parameters get modified as:

$$
R_{W}=R_{Z}=\sin (\beta-\alpha), \quad R_{b}=R_{\tau}=-\frac{\sin \alpha}{\cos \beta}, \quad R_{t}=\frac{\cos \alpha}{\sin \beta},
$$

where $\tan \beta$ is the usual ratio between the two Higgs boson vev, and the $\alpha$ is the usual angle that diagonalises the mass matrix of $\operatorname{Re}\left(H_{1}^{0}, H_{2}^{0}\right)$, with $\alpha \rightarrow \beta-\pi / 2$, in the decoupling limit. The angles $\alpha$ and $\beta$ depend on the model and specific deviations arise depending on how $m_{h} \approx 125 \mathrm{GeV}$ is reached: extra $D$-terms imply an increase in $h \rightarrow b \bar{b}$ while extra $F$-terms lead to a decrease (unless extra singlets are light) [121]. The total $R_{t}$ is the combination of the two effects discussed above.

As previously discussed, both the $W W^{*}$ and the $\gamma \gamma$ rates are roughly proportional to $R_{W}^{2}$; thereby this correlation prevents to go in the direction favoured by data (lower $W W^{*}$ and higher $\gamma \gamma$ ), as already observed in the context of numerical MSSM scans [58, 83-104], and in extensions of the MSSM [105-113].

\subsection{Dark matter models}

The main motivation for an invisible Higgs boson decay width comes from the existence of Dark Matter (DM) of the Universe. The Higgs portal [122] offers a natural possibility to 
couple the Higgs sector to the dark matter. If the dark matter particles are two times lighter than the Higgs boson, they can lead to invisible Higgs boson width. Because Higgs boson decays to fermion dark matter are essentially ruled out by direct detection constraints [59], in this scenario the dark matter is naturally scalar.

Let us consider, for example, the simplest DM model obtained adding to the SM a real singlet scalar field $S$ coupled to the Higgs doublet $H$ by the $-\lambda S^{2}|H|^{2}$ Lagrangian term $[123,124]$. Fixing the DM/Higgs boson coupling $\lambda$ assuming that the thermal relic $\mathrm{DM}$ abundance is equal to its cosmologically measured value $\Omega_{\mathrm{DM}}=0.112 \pm 0.0056$ [125] allows us to predict the Higgs boson invisible decay width and the direct DM detection cross section $\sigma_{\mathrm{SI}}$

$$
\Gamma(h \rightarrow S S)=\frac{\lambda^{2} V^{2}}{8 \pi m_{h}} \sqrt{1-4 \frac{M_{\mathrm{DM}}^{2}}{m_{h}^{2}}}, \quad \sigma_{\mathrm{SI}}=\frac{\lambda^{2} m_{N}^{4} f^{2}}{\pi M_{\mathrm{DM}}^{2} m_{h}^{4}} .
$$

The bound $\mathrm{BR}_{\text {inv }}<0.4$ at $95 \%$ C.L. derived in section 3.2 then implies $M_{\mathrm{DM}}>50 \mathrm{GeV}$ and $\sigma_{\mathrm{SI}}<0.410^{-44} \mathrm{~cm}^{2}$, assuming the nucleon matrix element $f=0.3$. While Higgs boson invisible decays to fermionic dark matter are already disfavoured, our work shows that also light scalar dark matter is not supported by data.

\subsection{Higgs boson or radion?}

The Higgs boson couples to the SM fermions with a strength proportional to fermion masses. Similar couplings can be obtained by considering an hypothetical particle $\varphi$, a radion, with a coupling to the trace of the SM energy-momentum tensor suppressed by some scale $\Lambda$ :

$$
\frac{\varphi}{\Lambda} T_{\mu}^{\mu}=\frac{\varphi}{\Lambda}\left(\sum_{f} m_{f} \bar{f} f-M_{Z}^{2} Z_{\mu}^{2}-2 M_{W}^{2} W_{\mu}^{2}+A\right) .
$$

In our language this is described by setting

$$
R \equiv R_{W}=R_{Z}=R_{t}=R_{b}=R_{\tau}=\sqrt{2} v / \Lambda,
$$

where $v=174 \mathrm{GeV}$. One important difference arises at quantum level: scale invariance is anomalous, such that a new term appears,

$$
A=-7 \frac{\alpha_{3}}{8 \pi} G_{\mu \nu}^{a} G_{\mu \nu}^{a}+\frac{11}{3} \frac{\alpha_{\mathrm{em}}}{8 \pi} F_{\mu \nu} F_{\mu \nu}
$$

where the numerical coefficients are the SM $\beta$-function coefficients for the strong and electromagnetic gauge couplings. As a result, the $\varphi$ decay widths into $g g$ and $\gamma \gamma$ differ from the corresponding Higgs boson decay widths [126-129].

Such a particle is often called 'radion' because it arises in the context of models with one warped extra dimension as the mode that controls its size. However, in this kind of models the radion can appear with extra couplings and together with other unseen particles.

We focus on the effective coupling in eq. (4.4), and find that the excess seen around $125 \mathrm{GeV}$ could be due to such a 'radion' rather than to the Higgs boson. The best fit is obtained at $R=0.28 \pm 0.03$ (i.e. $\Lambda \approx 870 \mathrm{GeV}$ ) and its quality is slightly worse that the best Higgs fit, as illustrated in figure 2. More data are needed to discriminate among the two possibilities. 


\section{Conclusions}

We performed a global phenomenological analysis of all Higgs boson collider data available after Moriond 2012 (including those presented in the context of fermiophobic Higgs boson searches) assuming that the hints observed at $m_{h} \approx 125 \mathrm{GeV}$ arise from the Higgs boson. The SM provides an acceptable fit, however it is not favoured: present data with large uncertainties favour a $h \rightarrow \gamma \gamma$ rate enhanced by a factor of $\approx 4$ and a $g g \rightarrow h$ rate reduced by a factor of 0.3. An invisible Higgs boson branching ratio larger than 0.4 is disfavoured, putting constraints on models where dark matter couples to the Higgs boson. Pure fermiophobic Higgs boson scenario gives almost as good fit as the SM but with significantly different predictions for the Higgs boson phenomenology. Partially fermiophobic scenarios are among those giving the best global fit. We find that the apparent excess can alternatively be interpreted as a 'radion' i.e. a particle similar to the Higgs boson, but coupled to the trace of the SM energy momentum tensor.

More LHC data should clarify whether the present anomalies in data are statistical fluctuations or first evidence of physics beyond the SM.

\section{Acknowledgments}

We thank Emidio Gabrielli and Christophe Grojean for discussions and Andrey Korytov and Bill Murray for useful communication. This work was supported by the ESF grants 8090, 8943, MTT8, MTT60, MJD140 by the recurrent financing SF0690030s09 project and by the European Union through the European Regional Development Fund.

Open Access. This article is distributed under the terms of the Creative Commons Attribution License which permits any use, distribution and reproduction in any medium, provided the original author(s) and source are credited.

\section{References}

[1] F. Englert and R. Brout, Broken symmetry and the mass of gauge vector mesons, Phys. Rev. Lett. 13 (1964) 321 [INSPIRE].

[2] P.W. Higgs, Broken symmetries, massless particles and gauge fields, Phys. Lett. 12 (1964) 132 [INSPIRE].

[3] P.W. Higgs, Broken symmetries and the masses of gauge bosons, Phys. Rev. Lett. 13 (1964) 508 [INSPIRE].

[4] G. Guralnik, C. Hagen and T. Kibble, Global conservation laws and massless particles, Phys. Rev. Lett. 13 (1964) 585 [InSPIRE].

[5] A. Djouadi, The anatomy of electro-weak symmetry breaking. I: the Higgs boson in the standard model, Phys. Rept. 457 (2008) 1 [hep-ph/0503172] [INSPIRE].

[6] F. Gianotti, Update on the standard model Higgs searches in ATLAS, CERN public seminar, December 13, Switzerland (2011).

[7] G. Tonelli, Update on the standard model Higgs searches in CMS, CERN public seminar, December 13, Switzerland (2011). 
[8] ATLAS collaboration, G. Aad et al., Combined search for the standard model Higgs boson using up to $4.9 \mathrm{fb}^{-1}$ of pp collision data at $\sqrt{\mathrm{s}}=7 \mathrm{TeV}$ with the ATLAS detector at the LHC, Phys. Lett. B 710 (2012) 49 [arXiv:1202.1408] [INSPIRE].

[9] CMS collaboration, S. Chatrchyan et al., Combined results of searches for the standard model Higgs boson in pp collisions at $\sqrt{s}=7 \mathrm{TeV}$, Phys. Lett. B $\mathbf{7 1 0}$ (2012) 26 [arXiv:1202.1488] [INSPIRE].

[10] E. Gabrielli, B. Mele and M. Raidal, Has a fermiophobic Higgs boson been detected at the LHC?, arXiv: 1202.1796 [INSPIRE].

[11] LHC Higgs Cross Section working group, S. Dittmaier et al., Handbook of LHC Higgs cross sections: 2. Differential distributions, arXiv:1201.3084 [CERN-2012-002] [INSPIRE].

[12] LHC Higgs Cross SeCtion working group, Branching ratios and partial-decay widths, https://twiki.cern.ch/twiki/bin/view/LHCPhysics/CERNYellowReportPageBR

[13] LHC Higgs Cross Section working group, S. Dittmaier et al., Handbook of LHC Higgs cross sections: 1. Inclusive observables, arXiv:1101.0593 [CERN-2011-002] [INSPIRE].

[14] LHC Higgs Cross Section working group, Recommended values on SM Higgs XS at $7 \mathrm{TeV}$, https://twiki.cern.ch/twiki/bin/view/LHCPhysics/CERNYellowReportPageAt7TeV.

[15] M. Dührssen et al., Extracting Higgs boson couplings from CERN LHC data, Phys. Rev. D 70 (2004) 113009 [hep-ph/0406323] [INSPIRE].

[16] R. Lafaye, T. Plehn, M. Rauch, D. Zerwas and M. Dührssen, Measuring the Higgs sector, JHEP 08 (2009) 009 [arXiv:0904.3866] [InSPIRE].

[17] A. Azatov, R. Contino and J. Galloway, Model-independent bounds on a light Higgs, JHEP 04 (2012) 127 [arXiv: 1202.3415] [INSPIRE].

[18] D. Carmi, A. Falkowski, E. Kuflik and T. Volansky, Interpreting LHC Higgs results from natural new physics perspective, arXiv:1202.3144 [INSPIRE].

[19] J. Espinosa, C. Grojean, M. Muhlleitner and M. Trott, Fingerprinting Higgs suspects at the LHC, JHEP 05 (2012) 097 [arXiv: 1202 .3697] [INSPIRE].

[20] M. Farina et al., Implications of XENON100 and LHC results for Dark Matter models, Nucl. Phys. B 853 (2011) 607 [arXiv: 1104.3572] [INSPIRE].

[21] CDF and D0 collaboration, Combined CDF and D0 searches for standard model Higgs boson production, arXiv:1203.3774 [INSPIRE].

[22] W. Fisher, New results from the Tevatron experiment, talk given at the Rencontres de Moriond, March 3-10, La Thuile, Italy (2012).

[23] CMS collaboration, Search for Higgs boson in VH Production with $H$ to bb, PAS-HIG-11-031 (2011).

[24] ATLAS collaboration, Search for the standard model Higgs boson produced in association with a vector boson and decaying to a b-quark pair using up to $4.7 \mathrm{fb}^{-1}$ of $\mathrm{pp}$ collision data at $\sqrt{s}=7 \mathrm{TeV}$ with the ATLAS detector at the LHC, ATLAS-CONF-2012-015 (2012).

[25] CMS collaboration, Combination of SM, SM4, FP Higgs boson searches, PAS-HIG-12-008 (2012).

[26] CMS collaboration, Search for the Higgs boson in the fully leptonic $W^{+} W^{-}$final state, PAS-HIG-11-024 (2011).

[27] ATLAS collaboration, Search for the standard model Higgs boson in the $H \rightarrow W W \rightarrow \ell \nu \ell \nu$ decay mode with $4.7 \mathrm{fb}^{-1}$ of ATLAS data at $\sqrt{s}=7 \mathrm{TeV}$, ATLAS-CONF-2012-012 (2012). 
[28] S. Kortner, SM scalar boson search with the ATLAS detector, talk given at the Rencontres de Moriond, March 3-10, La Thuile, Italy (2012).

[29] CMS Collaboration, Search for a standard model Higgs boson produced in the decay channel $H \rightarrow Z Z^{*} \rightarrow 4 \ell$, arXiv: 1202.1997 [INSPIRE].

[30] CMS collaboration, S. Chatrchyan et al., Search for the standard model Higgs boson decaying into two photons in pp collisions at $\sqrt{s}=7 \mathrm{TeV}$, Phys. Lett. B $\mathbf{7 1 0}(2012) 403$ [arXiv: 1202.1487] [INSPIRE].

[31] CMS collaboration, A search using multivariate techniques for a standard model Higgs boson decaying into two photons, CMS-PAS-HIG-12-001 (2012).

[32] ATLAS collaboration, G. Aad et al., Search for the standard model Higgs boson in the diphoton decay channel with $4.9 \mathrm{fb}^{-1}$ of pp collisions at $\sqrt{\mathrm{s}}=7 \mathrm{TeV}$ with ATLAS, Phys. Rev. Lett. 108 (2012) 111803 [arXiv:1202.1414] [INSPIRE].

[33] TEVNPH, CDF, D0 collaboration, Combined CDF and D0 search for standard model Higgs boson production with up to $10.0 \mathrm{fb}^{-1}$ of data, arXiv:1203.3774 [INSPIRE].

[34] CMS collaboration, Search for the fermiophobic model Higgs boson decaying into two photons, PAS-HIG-12-002 (2012).

[35] S. Dasu, Higgs sector beyond standard model, talk given at the Rencontres de Moriond, March 3-10, La Thuile, Italy (2012).

[36] ATLAS collaboration, Search for a fermiophobic Higgs boson in the diphoton decay channel with $4.9 \mathrm{fb}^{-1}$ of ATLAS data at $\sqrt{\mathrm{s}}=7 \mathrm{TeV}$, ATLAS-CONF-2012-013 (2012).

[37] T. Sjöstrand, S. Mrenna and P. Skands, A brief introduction to PYTHIA 8.1, Comput. Phys. Commun. 178 (2008) 852 [arXiv:0710.3820] [INSPIRE].

[38] J. Alwall, M. Herquet, F. Maltoni, O. Mattelaer and T. Stelzer, MadGraph 5: going beyond, JHEP 06 (2011) 128 [arXiv:1106.0522] [INSPIRE].

[39] CMS collaboration, S. Chatrchyan et al., Search for neutral Higgs bosons decaying to $\tau$ pairs in pp collisions at $\sqrt{s}=7 \mathrm{TeV}$, Phys. Lett. B 713 (2012) 68 [arXiv:1202.4083] [InSPIRE].

[40] ATLAS collaboration, Search for the Standard Model Higgs boson in the $H \rightarrow \tau^{+} \tau^{-}$decay mode with $4.7 \mathrm{fb}^{-1}$ of ATLAS data at $7 \mathrm{TeV}$, ATLAS-CONF-2012-014 (2012).

[41] O.J. Eboli and D. Zeppenfeld, Observing an invisible Higgs boson, Phys. Lett. B 495 (2000) 147 [hep-ph/0009158] [INSPIRE].

[42] R.M. Godbole, M. Guchait, K. Mazumdar, S. Moretti and D.P. Roy, Search for 'invisible' Higgs signals at LHC via associated production with gauge bosons, Phys. Lett. B 571 (2003) 184 [hep-ph/0304137] [INSPIRE].

[43] J.F. Kamenik and C. Smith, Could a light Higgs boson illuminate the dark sector?, arXiv: 1201.4814 [INSPIRE].

[44] M. Raidal and A. Strumia, Hints for a non-standard Higgs boson from the LHC, Phys. Rev. D 84 (2011) 077701 [arXiv: 1108.4903] [INSPIRE].

[45] Y. Mambrini, Higgs searches and singlet scalar dark matter: Combined constraints from XENON 100 and the LHC, Phys. Rev. D 84 (2011) 115017 [arXiv:1108.0671] [INSPIRE].

[46] A. Arhrib, R. Benbrik and N. Gaur, $H \rightarrow \gamma \gamma$ in inert Higgs doublet model, Phys. Rev. D 85 (2012) 095021 [arXiv:1201.2644] [inSPIRE].

[47] X.-G. He, B. Ren and J. Tandean, Hints of standard model Higgs boson at the LHC and light dark matter searches, Phys. Rev. D 85 (2012) 093019 [arXiv:1112.6364] [INSPIRE]. 
[48] C. Cheung and Y. Nomura, Higgs descendants, arXiv:1112.3043 [INSPIRE].

[49] X. Chu, T. Hambye and M.H. Tytgat, The four basic ways of creating dark matter through a portal, JCAP 05 (2012) 034 [arXiv: 1112.0493] [INSPIRE].

[50] O. Lebedev, H.M. Lee and Y. Mambrini, Vector Higgs-portal dark matter and the invisible Higgs, Phys. Lett. B 707 (2012) 570 [arXiv:1111.4482] [INSPIRE].

[51] C. Englert, J. Jaeckel, E. Re and M. Spannowsky, Evasive Higgs maneuvers at the LHC, Phys. Rev. D 85 (2012) 035008 [arXiv:1111.1719] [INSPIRE].

[52] I. Low, P. Schwaller, G. Shaughnessy and C.E. Wagner, The dark side of the Higgs boson, Phys. Rev. D 85 (2012) 015009 [arXiv:1110.4405] [InSPIRE].

[53] M. Pospelov and A. Ritz, Higgs decays to dark matter: beyond the minimal model, Phys. Rev. D 84 (2011) 113001 [arXiv:1109.4872] [INSPIRE].

[54] T. Cohen, J. Kearney, A. Pierce and D. Tucker-Smith, Singlet-doublet dark matter, Phys. Rev. D 85 (2012) 075003 [arXiv:1109.2604] [INSPIRE].

[55] X.-G. He and J. Tandean, Hidden Higgs boson at the LHC and light dark matter searches, Phys. Rev. D 84 (2011) 075018 [arXiv:1109.1277] [inSPIRE].

[56] C.-S. Chen and Y. Tang, Vacuum stability, neutrinos and dark matter, JHEP 04 (2012) 019 [arXiv: 1202 .5717] [INSPIRE].

[57] V. Barger, M. Ishida and W.-Y. Keung, Total width of 125 GeV Higgs, arXiv: 1203.3456 [INSPIRE].

[58] J. Cao, Z. Heng, J.M. Yang and J. Zhu, Higgs decay to dark matter in low energy SUSY: is it detectable at the LHC?, arXiv:1203.0694 [INSPIRE].

[59] A. Djouadi, O. Lebedev, Y. Mambrini and J. Quevillon, Implications of LHC searches for Higgs-portal dark matter, Phys. Lett. B 709 (2012) 65 [arXiv:1112.3299] [INSPIRE].

[60] G. Giudice, C. Grojean, A. Pomarol and R. Rattazzi, The strongly-interacting light Higgs, JHEP 06 (2007) 045 [hep-ph/0703164] [InSPIRE].

[61] R. Contino, C. Grojean, M. Moretti, F. Piccinini and R. Rattazzi, Strong double Higgs production at the LHC, JHEP 05 (2010) 089 [arXiv:1002.1011] [INSPIRE].

[62] R. Grober and M. Muhlleitner, Composite Higgs boson pair production at the LHC, JHEP 06 (2011) 020 [arXiv: 1012.1562] [INSPIRE].

[63] S. De Curtis, M. Redi and A. Tesi, The 4D composite Higgs, JHEP 04 (2012) 042 [arXiv:1110.1613] [INSPIRE].

[64] A. Strumia and F. Vissani, Neutrino masses and mixings and..., hep-ph/0606054 [INSPIRE].

[65] J. Basdevant, E.L. Berger, D. Dicus, C. Kao and S. Willenbrock, Final state interaction of longitudinal vector bosons, Phys. Lett. B 313 (1993) 402 [hep-ph/9211225] [INSPIRE].

[66] V.D. Barger, N.G. Deshpande, J.L. Hewett and T.G. Rizzo, A separate Higgs?, hep-ph/9211234 [INSPIRE].

[67] P. Bamert and Z. Kunszt, Gauge boson masses dominantly generated by Higgs triplet contributions?, Phys. Lett. B 306 (1993) 335 [hep-ph/9303239] [INSPIRE].

[68] H. Pois, T.J. Weiler and T.C. Yuan, Higgs boson decay to four fermions including a single top quark below $t \bar{t}$ threshold, Phys. Rev. D 47 (1993) 3886 [hep-ph/9303277] [INSPIRE].

[69] A. Stange, W.J. Marciano and S. Willenbrock, Higgs bosons at the Fermilab Tevatron, Phys. Rev. D 49 (1994) 1354 [hep-ph/9309294] [INSPIRE]. 
[70] M.A. Diaz and T.J. Weiler, Decays of a fermiophobic Higgs, hep-ph/9401259 [INSPIRE].

[71] A. Akeroyd, Fermiophobic Higgs bosons at the Tevatron, Phys. Lett. B 368 (1996) 89 [hep-ph/9511347] [INSPIRE].

[72] E. Gabrielli and B. Mele, Testing effective Yukawa couplings in Higgs searches at the Tevatron and LHC, Phys. Rev. D 82 (2010) 113014 [Erratum ibid. D 83 (2011) 079901] [arXiv: 1005.2498] [INSPIRE].

[73] E. Gabrielli and B. Mele, Effective Yukawa couplings and flavor-changing Higgs boson decays at linear colliders, Phys. Rev. D 83 (2011) 073009 [arXiv:1102.3361] [INSPIRE].

[74] E. Gabrielli and B. Mele, A radiatively improved fermiophobic Higgs boson scenario, arXiv:1112.5993 [INSPIRE].

[75] H. Haber, G.L. Kane and T. Sterling, The fermion mass scale and possible effects of Higgs bosons on experimental observables, Nucl. Phys. B 161 (1979) 493 [InSPIRE].

[76] A. Akeroyd, Fermiophobic and other nonminimal neutral Higgs bosons at the LHC, J. Phys. $G$ G 24 (1998) 1983 [hep-ph/9803324] [INSPIRE].

[77] G.F. Giudice and O. Lebedev, Higgs-dependent Yukawa couplings, Phys. Lett. B 665 (2008) 79 [arXiv: 0804.1753] [INSPIRE].

[78] K. Kannike, M. Raidal, D.M. Straub and A. Strumia, Anthropic solution to the magnetic muon anomaly: the charged see-saw, JHEP 02 (2012) 106 [arXiv:1111.2551] [INSPIRE].

[79] A. Falkowski, Pseudo-goldstone Higgs production via gluon fusion, Phys. Rev. D 77 (2008) 055018 [arXiv: 0711.0828] [INSPIRE].

[80] I. Low and A. Vichi, On the production of a composite Higgs boson, Phys. Rev. D 84 (2011) 045019 [arXiv: 1010.2753] [INSPIRE].

[81] A. Azatov and J. Galloway, Light custodians and Higgs physics in composite models, Phys. Rev. D 85 (2012) 055013 [arXiv: 1110.5646] [INSPIRE].

[82] A. Falkowski, S. Rychkov and A. Urbano, What if the Higgs couplings to $W$ and $Z$ bosons are larger than in the standard model?, JHEP 04 (2012) 073 [arXiv:1202.1532] [INSPIRE].

[83] L.J. Hall, D. Pinner and J.T. Ruderman, A natural SUSY Higgs near 126 GeV, JHEP 04 (2012) 131 [arXiv:1112.2703] [INSPIRE].

[84] H. Baer, V. Barger and A. Mustafayev, Implications of a $125 \mathrm{GeV}$ Higgs scalar for LHC SUSY and neutralino dark matter searches, Phys. Rev. D 85 (2012) 075010 [arXiv: 1112.3017] [INSPIRE].

[85] S. Heinemeyer, O. Stal and G. Weiglein, Interpreting the LHC Higgs search results in the MSSM, Phys. Lett. B 710 (2012) 201 [arXiv:1112.3026] [InSPIRE].

[86] A. Arbey, M. Battaglia, A. Djouadi, F. Mahmoudi and J. Quevillon, Implications of a 125 GeV Higgs for supersymmetric models, Phys. Lett. B 708 (2012) 162 [arXiv:1112.3028] [INSPIRE].

[87] A. Arbey, M. Battaglia and F. Mahmoudi, Constraints on the MSSM from the Higgs sector: a pMSSM study of Higgs searches, $B_{s}^{0} \rightarrow \mu^{+} \mu^{-}$and dark matter direct detection, Eur. Phys. J. C 72 (2012) 1906 [arXiv:1112.3032] [InSPIRE].

[88] M. Carena, S. Gori, N.R. Shah and C.E. Wagner, A 125 GeV SM-like Higgs in the MSSM and the $\gamma \gamma$ rate, JHEP 03 (2012) 014 [arXiv:1112.3336] [INSPIRE].

[89] P. Draper, P. Meade, M. Reece and D. Shih, Implications of a $125 \mathrm{GeV}$ Higgs for the MSSM and low-scale SUSY breaking, Phys. Rev. D 85 (2012) 095007 [arXiv:1112.3068] [INSPIRE]. 
[90] M. Kadastik, K. Kannike, A. Racioppi and M. Raidal, Implications of the 125 GeV Higgs boson for scalar dark matter and for the CMSSM phenomenology, JHEP 05 (2012) 061 [arXiv:1112.3647] [INSPIRE].

[91] O. Buchmueller et al., Higgs and supersymmetry, arXiv:1112.3564 [INSPIRE].

[92] J. Cao, Z. Heng, D. Li and J.M. Yang, Current experimental constraints on the lightest Higgs boson mass in the constrained MSSM, Phys. Lett. B 710 (2012) 665 [arXiv:1112.4391] [INSPIRE].

[93] A. Arvanitaki and G. Villadoro, A non standard model Higgs at the LHC as a sign of naturalness, JHEP 02 (2012) 144 [arXiv:1112.4835] [INSPIRE].

[94] Z. Kang, J. Li and T. Li, On naturalness of the (N)MSSM, arXiv:1201.5305.

[95] K.A. Olive, The impact of XENON100 and the LHC on supersymmetric dark matter, arXiv:1202.2324 [INSPIRE].

[96] J. Ellis and K.A. Olive, Revisiting the Higgs mass and dark matter in the CMSSM, Eur. Phys. J. C 72 (2012) 2005 [arXiv: 1202.3262] [INSPIRE].

[97] H. Baer, V. Barger and A. Mustafayev, Neutralino dark matter in mSUGRA/CMSSM with a $125 \mathrm{GeV}$ light Higgs scalar, JHEP 05 (2012) 091 [arXiv: 1202.4038] [INSPIRE].

[98] N. Desai, B. Mukhopadhyaya and S. Niyogi, Constraints on invisible Higgs decay in MSSM in the light of diphoton rates from the LHC, arXiv:1202.5190 [INSPIRE].

[99] J. Cao, Z. Heng, J.M. Yang, Y. Zhang and J. Zhu, A SM-like Higgs near $125 \mathrm{GeV}$ in low energy SUSY: a comparative study for MSSM and NMSSM, JHEP 03 (2012) 086 [arXiv: 1202.5821] [INSPIRE].

[100] F. Jegerlehner, Implications of low and high energy measurements on SUSY models, arXiv: 1203.0806 [INSPIRE].

[101] Z. Kang, T. Li, T. Liu, C. Tong and J.M. Yang, A heavy SM-like higgs and a light stop from Yukawa-deflected gauge mediation, arXiv:1203.2336 [INSPIRE].

[102] D. Curtin, P. Jaiswal and P. Meade, Excluding electroweak baryogenesis in the MSSM, arXiv:1203.2932 [INSPIRE].

[103] N.D. Christensen, T. Han and S. Su, MSSM Higgs bosons at the LHC, arXiv:1203.3207 [INSPIRE].

[104] F. Boudjema and G.D. La Rochelle, BMSSM higgses at $125 \mathrm{GeV}$, arXiv:1203.3141.

[105] T. Moroi, R. Sato and T.T. Yanagida, Extra matters decree the relatively heavy Higgs of mass about $125 \mathrm{GeV}$ in the supersymmetric model, Phys. Lett. B 709 (2012) 218 [arXiv: 1112.3142] [INSPIRE].

[106] U. Ellwanger, A Higgs boson near $125 \mathrm{GeV}$ with enhanced di-photon signal in the NMSSM, JHEP 03 (2012) 044 [arXiv:1112.3548] [inSPIRE].

[107] M. Gozdz, Lightest Higgs boson masses in the R-parity violating supersymmetry, arXiv: 1201.0875 [INSPIRE].

[108] J.F. Gunion, Y. Jiang and S. Kraml, The constrained NMSSM and Higgs near $125 \mathrm{GeV}$, Phys. Lett. B 710 (2012) 454 [arXiv:1201.0982] [inSPIRE].

[109] S. King, M. Muhlleitner and R. Nevzorov, NMSSM Higgs benchmarks near 125 GeV, Nucl. Phys. B 860 (2012) 207 [arXiv:1201.2671] [INSPIRE].

[110] T.G. Rizzo, Gauge kinetic mixing in the $E_{6} S S M$, Phys. Rev. D 85 (2012) 055010 [arXiv: 1201.2898] [INSPIRE]. 
[111] Z. Kang, J. Li and T. Li, On naturalness of the (N)MSSM, arXiv:1201.5305 [INSPIRE].

[112] C.-F. Chang, K. Cheung, Y.-C. Lin and T.-C. Yuan, Mimicking the standard model Higgs boson in UMSSM, arXiv:1202.0054 [INSPIRE].

[113] D.A. Vasquez et al., The $125 \mathrm{GeV}$ Higgs in the NMSSM in light of LHC results and astrophysics constraints, arXiv:1203.3446 [INSPIRE].

[114] A. Djouadi, Squark effects on Higgs boson production and decay at the LHC, Phys. Lett. B 435 (1998) 101 [hep-ph/9806315] [INSPIRE].

[115] ATLAS collaboration, Search for scalar top quark pair production in natural gauge mediated supersymmetry models with the ATLAS detector in pp collisions at $\sqrt{s}=7 \mathrm{TeV}$, ATLAS-CONF-2012-036 (2012).

[116] ATLAS collaboration, Search for supersymmetry in pp collisions at $\sqrt{s}=7 \mathrm{TeV}$ in final states with missing transverse momentum and b-jets with the ATLAS detector, ATLAS-CONF-2012-003 (2012).

[117] ATLAS collaboration, Search for gluinos in events with two same-sign leptons, jets and missing transverse momentum with the ATLAS detector in pp collisions at $\sqrt{s}=7 \mathrm{TeV}$, ATLAS-CONF-2012-004 (2012).

[118] ATLAS collaboration, Hunt for new phenomena using large jet multiplicities and missing transverse momentum with ATLAS in $L=4.7 \mathrm{fb}^{-1}$ of $\sqrt{\mathrm{s}}=7 \mathrm{TeV}$ proton-proton collisions, ATLAS-CONF-2012-037 (2012).

[119] CMS collaboration, Search for supersymmetry in all-hadronic events with $\alpha_{T}$, PAS-SUS-11-003 (2011).

[120] ATLAS collaboration, A. Marzin, Searches for third generation SUSY in ATLAS, arXiv: 1205.3885 [INSPIRE].

[121] K. Blum, R.T. D'Agnolo and J. Fan, Natural SUSY predicts: Higgs couplings, in preparation.

[122] B. Patt and F. Wilczek, Higgs-field portal into hidden sectors, hep-ph/0605188 [INSPIRE].

[123] J. McDonald, Gauge singlet scalars as cold dark matter, Phys. Rev. D 50 (1994) 3637 [hep-ph/0702143] [INSPIRE].

[124] C. Burgess, M. Pospelov and T. ter Veldhuis, The minimal model of nonbaryonic dark matter: a singlet scalar, Nucl. Phys. B 619 (2001) 709 [hep-ph/0011335] [INSPIRE].

[125] D. Larson et al., Seven-year Wilkinson Microwave Anisotropy Probe (WMAP) observations: power spectra and WMAP-derived parameters, Astrophys. J. Suppl. 192 (2011) 16 [arXiv: 1001.4635$]$ [INSPIRE].

[126] L. Randall and R. Sundrum, A large mass hierarchy from a small extra dimension, Phys. Rev. Lett. 83 (1999) 3370 [hep-ph/9905221] [INSPIRE].

[127] Y. Eshel, S.J. Lee, G. Perez and Y. Soreq, Shining flavor and radion phenomenology in warped extra dimension, JHEP 10 (2011) 015 [arXiv: 1106.6218] [INSPIRE].

[128] V. Barger and M. Ishida, Randall-Sundrum reality at the LHC, Phys. Lett. B 709 (2012) 185 [arXiv: 1110.6452] [INSPIRE].

[129] K. Cheung and T.-C. Yuan, Could the excess seen at 124-126 GeV be due to the Randall-Sundrum radion?, Phys. Rev. Lett. 108 (2012) 141602 [arXiv:1112.4146] [INSPIRE]. 